\title{
Investigating developmental delay in South Africa: A pragmatic approach
}

\author{
K J Fieggen, ${ }^{1} \mathrm{MB}$ ChB, FCPaed (SA), Cert Medical Genetics (SA); \\ L A Lambie, ${ }^{2}$ MB BCh, FCPaed (SA), Cert Medical Genetics (SA), MSc Med (Genetic Counselling); \\ K A Donald, ${ }^{3} \mathrm{MB}$ ChB, MRPCH (UK), FCPaed (SA), MPhil (Paed Neuro), Cert Paediatric Neurology (SA), PhD
}

\begin{abstract}
${ }^{1}$ Division of Human Genetics, Department of Medicine, Faculty of Health Sciences, Groote Schuur Hospital and University of Cape Town, South Africa ${ }^{2}$ Genetics National Reference Laboratory, Ampath Trust, Pretoria, and Division of Human Genetics, School of Pathology, Faculty of Health Sciences, University of the Witwatersrand, Johannesburg, South Africa

${ }^{3}$ Division of Developmental Paediatrics, Department of Paediatrics and Child Health, Red Cross War Memorial Children's Hospital, Faculty of Health Sciences, University of Cape Town, South Africa
\end{abstract}

Corresponding author: KJ Fieggen (karen.fieggen@uct.ac.za)

\begin{abstract}
Global developmental delay and intellectual disability are common disorders in every population, with a prevalence of 1 - 5\%. Although a specific cause is not always immediately identifiable, for many affected people the aetiology is genetic or the result of secondary insult to the developing brain. Investigating children with intellectual developmental disorders (IDD) may be a significant challenge, especially in resourcelimited settings. Comprehensive clinical evaluation is the first step and frequently determines the direction of further investigations. Hearing and vision screening and thyroid function tests should be performed in most patients. Children with neurological findings should undergo brain imaging, and careful consideration should be given to potential metabolic disorders in all children with IDD, a number of whom are amenable to treatment. Most children in whom a specific direction is not suggested after history-taking and examination, should be tested for chromosomal abnormalities, ideally with chromosomal microarray, which has a diagnostic detection rate of 8 - $20 \%$ in IDD. Additional tests may be indicated in particular settings, including broad-based testing for single-gene disorders, such as next-generation sequencing gene panels or exome analysis. All genetic testing has the potential of finding variants of uncertain significance, particularly when there are limited population data, such as in Africa. Making a specific diagnosis in IDD is of benefit to patients, their families and society. Therefore, a rational approach to investigating this group of patients is essential to maximise the health benefit in a cost-effective way.
\end{abstract}

S Afr Med J 2019;109(4):210-213. DOI:10.7196/SAMJ.2019.v109i4.13991

Global developmental delay (GDD) and intellectual disability (ID) are both defined by delays or deficits in at least two domains of function, with onset during the developmental period. Typically, the term GDD is reserved for children $<5$ years of age, reflecting the challenges of formalised testing in young children. In contrast, a diagnosis of ID reflects deficits in cognitive function and a broad range of adaptive behaviours. ${ }^{[1]}$ These two groups of disorders may be grouped under the umbrella term intellectual developmental disorders (IDD). ${ }^{[2]}$ These disorders may be secondary to an external insult to the developing brain or primary, reflecting an early developmental difference. The focus of this approach is on the investigation of what may be defined as the IDD group of disorders, with the exclusion of the primary motor developmental disorders (such as cerebral palsy) and children presenting with progressive neurological disease or neuroregression, for whom a different diagnostic approach is indicated. The prevalence of IDD in South Africa (SA) is not well documented, but worldwide prevalence data suggest that $1-2 \%$ of the population may have an intellectual disability, with a prevalence of $2-5 \%$ for GDD. ${ }^{[3]}$ The real extent of the impact on function may be much greater, with population studies of early childhood development in a broad sample of 3 - 4-year-old children in 35 lowand middle-income countries (LMIC) finding that approximately a third failed to meet the expected cognitive and socioemotional milestones. ${ }^{[4]} \mathrm{A}$ study in rural SA found a minimum prevalence of ID in children of $3.56 \%{ }^{[5]}$
In SA, IDD may more commonly be caused, or further impacted on, by secondary exposures than in higher-income countries. These may include contributing factors such as prenatal alcohol exposure, ${ }^{[6]}$ congenital infections and HIV-related developmental disorders, ${ }^{[7]}$ which have been well documented as major contributors to the loss of developmental potential in SA children. There is, however, no reason to expect that primary genetic disorders of development are less common in SA than in better-resourced countries. Establishing the contributing aetiology(ies) or making a specific diagnosis in a child with IDD has numerous advantages. These include providing information on natural history and prognosis, avoiding additional costly and unnecessary investigations, refining treatment options, providing diagnosis-specific health surveillance and support, as well as providing information on recurrence risk for families. ${ }^{[8]}$ There is also good evidence to support the substantial value to parents of having a 'name' for their child's challenges. ${ }^{[9]}$ In the context of resource rationing in all health systems, but particularly the public healthcare system, the reality of the costs of specific diagnostic investigations has to be balanced with their intrinsic and comparative value to other investigations in terms of diagnostic yield and clinical use.

\section{Assessment of the child with an} intellectual developmental disorder

A comprehensive history and a thorough clinical examination are essential to make a diagnosis and to plan and interpret further 
special investigations that yield more diagnostic results when directed in this way. ${ }^{[10]}$ In particular, attention should be given to taking a complete pregnancy and birth/ perinatal history, including teratogen and congenital infection exposures, a three-generation family history (which should include direct questioning about pregnancy loss and other neurological and neurodevelopmental phenotypes), as well as documenting details of the course of the child's health, growth and development up to the time of evaluation. Physical examination should include charting all growth parameters (including head circumference), noting any dysmorphic features, a full systemic examination, a detailed neurological examination and developmental/cognitive assessment. Hearing screening should always be considered and vision assessed. ${ }^{[1]}$ Studies have shown that in up to a third of patients, a diagnosis can be reached or strongly suspected based on history and examination alone. ${ }^{[10]}$

This clinical process should yield three groups of patients. The first group includes those in whom a clinical diagnosis is made and investigations can be specifically tailored to confirm the diagnosis or investigate comorbidities, such as Down syndrome. There is a second group in whom the initial assessment leads to a suspected or possible diagnosis, e.g. a developmentally delayed child in whom findings of coarse features and organomegaly suggest a storage disorder. For this group of children, investigations will again be directed by clinical reasoning, but can be staged and directed by clinical context. The third group of children have unexplained IDD. In this group no specific clues to aetiology are evident, and a broader approach to further investigations is indicated. ${ }^{[11]}$ Fig. 1 illustrates a suggested approach to seeking a diagnosis in SA children with IDD.

\section{Investigations in}

\section{unexplained intellectual} developmental disorders Chromosomal abnormalities

Current international guidelines recommend chromosomal microarray (CMA) testing for chromosomal imbalance as the first investigation in unexplained ID or GDD. ${ }^{[3,8,11,12]}$ The diagnostic detection rate of CMA is reported as $8-20 \%$. This replaces the more traditional, but low-resolution, karyotype chromosomal analysis, which has a detection rate of $\sim 3 \%$, if the common aneuploidies are excluded. ${ }^{[13,14]}$ In general, higher detection rates are found in children who have additional features of atypical growth, structural congenital

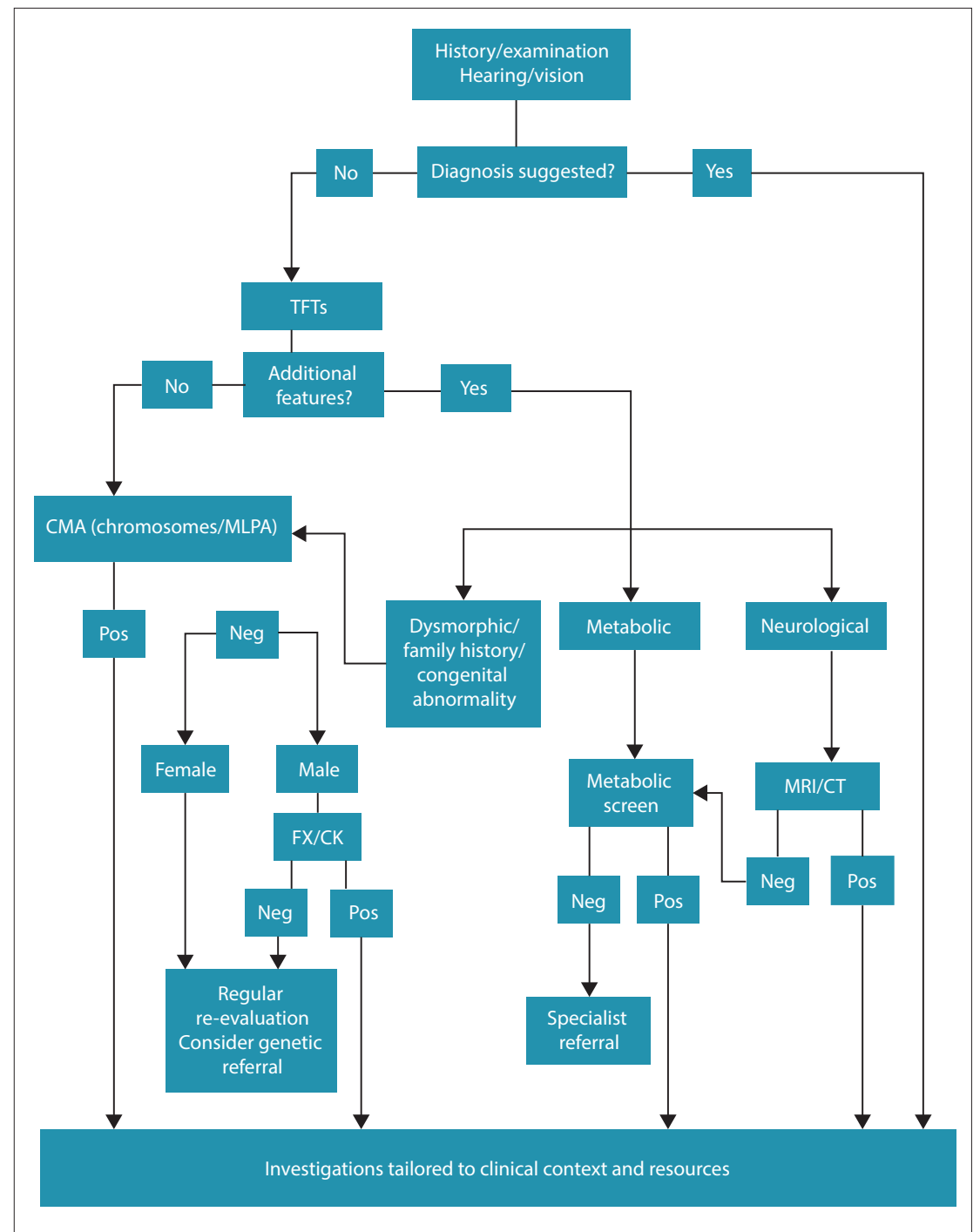

Fig. 1. Flow chart for investigating intellectual developmental disorders in children. $($ TFTs $=$ thyroid function tests; $C M A=$ chromosomal microarray; $M L P A=$ multiplex ligation-dependent probe amplification; pos = positive; neg = negative; $M R I=$ magnetic resonance imaging; $C T=$ computed tomography; $F X=$ fragile $X ; C K=$ creatine kinase.)

abnormalities, sensory impairments and/or dysmorphic features. It should be remembered, though, that CMA will not determine mechanism of dosage imbalance or detect chromosomal rearrangements that do not result in a net gain or loss of genetic material, and the resolution is limited depending on the specific CMA used. ${ }^{[13]}$ Conventional karyotype and supplementary cytogenetic techniques, such as fluorescence in situ hybridisation (FISH), retain a place in confirmation of certain CMA results, in determination of mechanism of chromosome imbalances, such as Down syndrome (which may inform recurrence risks), and in some cases of recurrent pregnancy loss. Multiplex ligation-dependent probe amplification (MLPA) is also a molecular technique designed to detect chromosomal imbalances. Commercial kits targeting areas of the genome well described to be prone to loss or gain and associated with developmental disorders are available. Although less costly individually, performing a karyotype and two MLPA kits are similar in cost to CMA, which has a much better resolution and hence diagnostic yield. Therefore, although CMA is the preferred test for chromosomal abnormalities, MLPA may have a place where the resources required for CMA are not available.

\section{Fragile $\mathrm{X}$ syndrome testing}

The clinical presentation of fragile $\mathrm{X}$ syndrome (FXS) can be nonspecific, particularly in young children, and in the past has prompted routine testing for FMR1 expansion mutations in IDD, mostly in boys, although the disorder also presents in girls. A systematic review and meta-analysis pub- 
lished in 2014 found the population frequency of full expansions to be 1.4/10 000 for males and 0.9/10 000 for females. ${ }^{[15]}$ Although still commonly performed as a first-line investigation in boys, it may not be cost effective in all children with IDD, but is definitely indicated in those with a suggestive family history or clinical features. Other $\mathrm{X}$-linked single-gene disorders are collectively more common than FXS, and in situations where X-linked inheritance is possible, additional genetic testing approaches need to be considered, as discussed below. ${ }^{[8]}$

\section{Neuroimaging}

As most young children (especially those with IDD) need an anaesthetic or sedation for neuroimaging, its role as a first-line investigation remains controversial in unexplained IDD. Children who have associated epilepsy, particularly if there is a focal element, those with neurological signs and those with a significant disturbance in head growth, should be considered for magnetic resonance imagimg (MRI) or, if not available, a computed tomography (CT) scan early in their diagnostic investigations. ${ }^{[11]}$ For most children with nonprogressive ID or GDD without additional neurological symptoms or physical findings, neuroimaging has a relatively low diagnostic yield.

\section{Metabolic investigations}

There is universal agreement that thyroid function tests should be included in the initial investigation of unexplained IDD, as hypothyroidism is amenable to treatment and testing is accessible and affordable. Indications for additional metabolic screening and testing in the absence of clinical clues are not quite as consistent. Some guidelines advocate for this approach in all children with IDD, with particular emphasis on disorders potentially amenable to treatment. ${ }^{[8,10]}$ Such investigations are costly and, given that there is usually some clinical indication to suggest a metabolic disorder, it may be reasonable to reserve these for selected cases, especially in resource-constrained environments. A retrospective study by Hart et al. ${ }^{[14]}$ showed that no metabolic disorders were identified in children for whom early developmental impairment was the only clinical feature. It does, however, underscore the attention to detail that needs to be elicited on history-taking and examination, such as parental consanguinity, a history of a fluctuant course, episodes of deterioration triggered by stress or additional clinical features suggesting a metabolic aetiology.

Tests that are most often included in a broad screen for metabolic aetiology of developmental delay include plasma lactate, ammonia, urine amino and organic acids and serum amino acids, along with routine biochemistry, such as urea and electrolytes and liver function tests. ${ }^{[3,4]}$ Other guidelines include serum homocysteine and acylcarnitines, as well as oligosaccharides, glycosaminoglycans and purines and pyrimidines in the first-line screening, with other metabolic tests being driven by clinical indication. ${ }^{[8]}$

\section{Nonspecific screening tests}

General screening tests, such as full blood count, urea and electrolytes, iron studies and lead levels, are often included in the initial investigation of IDD, but there is little evidence to suggest that they add value unless clinically directed, and may be omitted without compromising care. ${ }^{[14]}$ Creatinine kinase level determination is recommended in some guidelines as the screening test for Duchenne muscular dystrophy (DMD) in young boys with GDD, recognising that GDD may precede signs of muscle disease in some of these boys. As the test is inexpensive and carries significant management and counselling implications for the child and family, it is worth considering in this subgroup of children.

\section{Genetic testing strategies for single- gene disorders}

In children with IDD in whom a genetic diagnosis is suspected, it is important to select the most appropriate testing strategy and to be aware of the limitations of the test. Genetic mutations can be detected by various techniques. Targeted mutation testing for a single mutation or a limited number of mutations in a particular gene still retains a place as a cost-effective strategy for populations that have founder mutations or for disorders that are commonly caused by a small number of mutations. These strategies are, however, inherently limited to the ability to include, but not exclude, a diagnosis. Sanger sequencing of a specific gene has long been considered the gold standard for testing for single-gene disorders, but is not able to detect deletions or duplications and becomes expensive if sequential testing of a number of genes that may cause the phenotype in question. Next-generation sequencing (NGS) technologies allow rapid, reliable and simultaneous testing of multiple genes. Gene-panel testing using NGS, in which multiple genes implicated in disorders for which there is clinical and genetic heterogeneity and overlap, has replaced sequential single-gene testing as a quicker and more cost-effective strategy. ${ }^{[16]}$

In IDD, a large number of genes are implicated, with many as yet possibly undiscovered. This makes testing challenging, unless there are clinical clues to direct the clinician towards a single gene or panel of genes. To have a less directed approach in this context, broader molecular testing strategies are needed. Whole-exome sequencing (WES) analyses the majority of the protein-coding sequences of the genome for mutations. In contrast, whole-genome sequencing (WGS) analyses the whole genome, recognising that many variants that can potentially influence health may lie in regulatory or control regions of the genome. These strategies have the potential to detect mutations in genes known to be associated with IDD that were not clinically suspected, and to allow for new gene discovery. While WES has been used in diagnostic practice for a number of years and WGS is becoming available as a diagnostic test, these strategies remain hampered by the cost and complexity of interpretation, particularly in less-well-characterised populations, such as those in Africa. However, with diagnostic detection rates of up to $60 \%$ in selected IDD cases, ${ }^{[16,17]}$ these exome/genome wide approaches are likely to become more commonplace in the near future, but will continue to require comprehensive clinical correlation. ${ }^{[16]}$

\section{Interpreting genetic test results}

One of the challenges of genetic testing is the extent of normal human variation. It can therefore be challenging to determine if a detected variant is clinically and functionally significant. This is true for small chromosomal copy number changes, as well as variants within genes and their regulatory regions. As a result, a genetic test can produce three possible outcomes: ${ }^{[1]}$

- abnormal result with a pathogenic or likely pathogenic variant detected

- normal result with no clinically significant variant detected

- variant/s of uncertain significance detected.

Variants of uncertain significance cannot be used for decisionmaking, as there is insufficient evidence to determine if the change is pathogenic or benign. Further evidence (including, possibly, family segregation studies and functional analysis) may be needed before disease association can be assigned. As evidence accumulates over time, including knowledge regarding population-specific variation, reclassification of the significance of a variant is possible. This should be taken into consideration during longitudinal follow-up of patients and families. 


\section{Conclusion}

Patients with GDD and ID are present in all populations and present to healthcare services in all communities. A systematic clinical evaluation and appropriate investigations can contribute in many ways to improved healthcare for those affected, their families and society in general. Knowledge about the value, limitations and interpretation of investigations is core to delivering on the expectation of improved health and wellbeing that advances in the field of medical genetics can bring.

\section{Declaration. None.}

Acknowledgements. We acknowledge colleagues who shared their experience and opinions.

Author contributions. KJF wrote the manuscript after extensive consultation with and contributions from LAL and KAD.

\section{Funding. None.}

\section{Conflicts of interest. None.}

1. Pinchefsky E, Shevell M. Intellectual disabilities and global developmental delay. In: Goldstein S, deVries M, eds. Handbook of DSM-5 Disorders in Children and Adolescents. Berlin: Springer, 2017.

2. Carulla LS, Reed GM, Vaez-Azizi L, et al. Intellectual developmental disorders: Towards a new name, definition and framework for 'mental retardation/intellectual disability' in ICD-11. World Psychiat 2011;10(3):175-180. https://doi.org/10.1002/j.2051-5545.2011.tb00045.x

3. Mithyantha R, Kneen R, McCann E, Gladstone M. Current evidence-based recommendations on investigating children with global developmental delay. Arch Dis Child 2017;102(11):1071-1076. https:// doi.org/10.1136/archdischild-2016-31127

4. McCoy DC, Peet ED, Ezzati M, et al. Early childhood developmental status in low- and middle-income countries: National, regional, and global prevalence estimates using predictive modeling. PLOS Med 2016;13(6):e1002034. https://doi.org/10.1371/journal.pmed.1002034
5. Christianson AL, Zwane ME, Manga P, et al. Children with intellectual disability in rural South Africa: Prevalence and associated disability. J Intellect Disabil Res 2002;46(2):179-186. https://doi.org/10.1046/ $1365-2788.2002 .00390 x$

6. Olivier L, Curfs LM, Viljoen DL. Fetal alcohol spectrum disorders: Prevalence in South Africa. S Afr Med J 2016;106(6 Suppl 1):S103-S106. https://doi.org/10.7196/SAMJ.2016.v106i6.11009

7. Donald KA, Walker KG, Kilborn T, et al. HIV encephalopathy: Pediatric case series description and insights from the clinic coalface. AIDS Res Ther 2015;12(1):2. https://doi.org/10.1186/s12981014-0042-7

8. Moeschler JB, Shevell M. Comprehensive evaluation of the child with intellectual disability or global developmental delays. Pediatrics 2014;134(3):e903-e918. https://doi.org/10.1542/peds.2014-1839

9. Makela NL, Birch PH, Friedman JM, Marra CA. Parental perceived value of a diagnosis for intellectual disability (ID): A qualitative comparison of families with and without a diagnosis for their child's ID. Am J Med Genet 2009;149A(11):2393-2402. https://doi.org/10.1002/ajmg.a.33050

10. Van Karnebeek CDM, Scheper FY, Abeling NG, et al. Etiology of mental retardation in children referred to a tertiary care center: A prospective study. Am J Ment Retard 2005;110(4):253-267.

11. O’Byrne JJ, Lynch SA, Treacy EP, et al. Unexplained developmental delay/learning disability: Guidelines for best practice protocol for first line assessment and genetic/metabolic/radiological investigations. Ir J Med Sci 2016;185(1):241. https://doi.org/10.1007/s1 1845-015-1284-7

12. Silove N, Collins F, Ellaway C. Investigation of developmental delay. J Paediatr Child Health 2013;49(7):519-525. https://doi.org/10.1111/jpc.12176

13. Miller DT, Adam MP, Aradhya S, et al. Consensus statement: Chromosomal microarray is a first-tier clinical diagnostic test for individuals with developmental disabilities or congenital anomalies. Am J Hum Genet 2010;86(5):749-764. https://doi.org/10.1016/j.jhh.2010.04.006

14. Hart AR, Sharma R, Atherton M, et al. Aetiological investigations in early developmental impairment: Are they worth it? Arch Dis Child 2017;102(11):1004-1013. https://doi.org/10.1136/archdischild2017-312843

15. Hunter J, Rivero-Arias O, Angelov A, Kim E, Fotheringham I, Leal J. Epidemiology of fragile X syndrome: A systematic review and meta-analysis. Am J Med Genet 2014;164A(7):1648-1658. https://doi. org/10.1002/ajmg.a.36511

16. Carvill GL, Mefford HC. Next-generation sequencing in intellectual disability. J Pediat Genet 2015;4(3):128135. https://doi.org/10.1055/s-0035-1564439

17. Wilfert AB, Sulovari A, Turner TN, Coe BP, Eichler EE. Recurrent de novo mutations in neurodevelopmental disorders: Properties and clinical implications. Genome Med 2017;9(1):101. https:// doi.org/10.1186/s13073-017-0498-x

Accepted 20 February 2019. 